\title{
Strong Completeness and Limited Canonicity for PDL
}

\author{
Gerard Renardel de Lavalette • Barteld Kooi • \\ Rineke Verbrugge
}

Published online: 29 September 2007

(C) Springer Science+Business Media B.V. 2007

\begin{abstract}
Propositional dynamic logic (PDL) is complete but not compact. As a consequence, strong completeness (the property $\Gamma \models \varphi \Rightarrow \Gamma \vdash \varphi$ ) requires an infinitary proof system. In this paper, we present a short proof for strong completeness of PDL relative to an infinitary proof system containing the rule from $\left[\alpha ; \beta^{n}\right] \varphi$ for all $n \in \mathbb{N}$, conclude $\left[\alpha ; \beta^{*}\right] \varphi$. The proof uses a universal canonical model, and it is generalized to other modal logics with infinitary proof rules, such as epistemic knowledge with common knowledge. Also, we show that the universal canonical model of PDL lacks the property of modal harmony, the analogue of the Truth lemma for modal operators.
\end{abstract}

Keywords Propositional dynamic logic · Strong completeness · Canonical model · Model disharmony

\section{Introduction}

Dynamic logic is a modal logic for reasoning about computer processes. This branch of logic was started by Pratt (1976) (with Salwicki's Algorithmic logic (Salwicki 1970) as a predecessor). The propositional part of dynamic logic (PDL) became an object of

G. Renardel de Lavalette $(\varangle)$

Department of Computing Science, University of Groningen, P.O. Box 407, Groningen 9700 AK,

The Netherlands

e-mail: g.r.renardel.de.lavalette@ rug.nl

B. Kooi

Faculty of Philosophy, University of Groningen, Groningen, The Netherlands

e-mail: b.p.Kooi@rug.nl

R. Verbrugge

Department of Artificial Intelligence, University of Groningen, Groningen, The Netherlands

e-mail: rineke@ai.rug.nl 
study in itself. Segerberg (1982) gave an axiomatization (see below) that was shown to be complete by several authors: the standard reference is Kozen and Parikh (1981). See also Harel (1984) and Harel et al. (2000) for surveys.

Strong completeness (also called extended completeness) is the following property of a logical system with derivability relation $\vdash$ and semantic entailment relation $\models$ :

$$
\Gamma \models \varphi \text { implies } \Gamma \vdash \varphi \text {, for all formulas } \varphi \text { and all sets of formulas } \Gamma \text {. }
$$

This generalizes weak completeness, where $\Gamma$ is empty. Observe that weak completeness implies strong completeness whenever the logic in question is semantically compact, i.e. when $\Gamma \models \varphi$ implies that there is a finite $\Gamma^{\prime} \subseteq \Gamma$ with $\Gamma^{\prime} \models \varphi$, hence $\models \bigwedge \Gamma^{\prime} \rightarrow \varphi$. This is, for example, the case in propositional and predicate logic, and in many modal logics such as $\mathrm{K}$ and S5.

Segerberg's axiomatization of PDL is only weakly complete, since PDL is not compact: we have that $\left\{\left[a^{n}\right] p \mid n \in \mathbb{N}\right\} \models\left[a^{*}\right] p$ but there is no natural number $k$ with $\left\{\left[a^{n}\right] p \mid n \leq k\right\} \models\left[a^{*}\right] p$. As a consequence, we do not have strong completeness for any finitary axiomatization, a fortiori not for its usual, weakly complete proof system (see Definition 3). So strong completeness requires an infinitary proof system. The first topic of this paper is a short proof of strong completeness for an extension of PDL with infinitary proof rules. The proof uses a universal canonical model in which every nonderivable sequent $\Gamma \vdash \varphi$ is refuted: this contrasts with the ordinary completeness proof for PDL (see e.g. Kozen and Parikh 1981 or Harel et al. 2000), which is based on finite canonical models for finite fragments of the language.

Several infinitary systems for PDL and related logics have been presented in the literature. Early examples are Propositional Algorithmic Logic by Mirkowska (1981) and Goldblatt (1982) about a logical system related to PDL. Both prove strong completeness, but the proofs are rather involved and do not transfer to $\mathrm{PDL}_{\omega}$.

In Knijnenburg (1988) and Knijnenburg and van Leeuwen (1991), an infinitary axiomatization of PDL is presented that is directly comparable with the logic $\mathrm{PDL}_{\omega}$ investigated here. However, the completeness results in these publications rely on Lindenbaum's lemma (every consistent set is contained in a maximal consistent set) for compact logics, whereas the logic in question is not compact. So, although we believe that the completeness results hold, we think that the proofs are not correct (as has been acknowledged by one of the authors in private communication).

In the related publications, Goldblatt (1993) and Segerberg (1994) investigate classes of infinitary logics obtained by adding countably many rules that satisfy certain properties to some basic proof system. They prove strong completeness for these logics with respect to appropriate classes of models, and show that $\mathrm{PDL}_{\omega}$ is an instance. The completeness proofs are rather complicated and use both maximal consistent sets and saturated sets (in the sense of Definition 5 below).

In Sect. 3 of the present paper, we give a rather short and straightforward proof of strong completeness for PDL $\omega$, based on the (hitherto undiscovered) identity of saturated and maximal consistent sets. It is inspired on the work of Goldblatt and Segerberg mentioned above. We shortly indicate how to generalize the proof method to other infinitary logics, and we show that several logics are canonical, in the sense that their axioms are valid in the frame underlying their canonical model. In Subsect. 3.1, 
we explain the problem in proving strong completeness for $\mathrm{PDL}_{\omega}$, and we also indicate why the Rasiowa-Sikorski lemma cannot help us here.

In Sect.4, we discuss a rather peculiar feature of the canonical model. In the standard completeness proof for a (modal) logic, the Truth lemma states that a formula is true in a world (i.e. a maximal consistent set) of the canonical model iff it is an element of that world. In other words: at the formula level, there is agreement between the semantics and the proof theoretical aspects of the canonical model. Therefore we call this property formula harmony, and its analogue for modal operators we call modal harmony. We show that, to our surprise, the canonical model of $\mathrm{PDL}_{\omega}$ fails to have modal harmony, while at the same time it does have formula harmony. We first prove a similar disharmony result for ancestral logic (the logic with modalities $\square$ and its reflexive transitive closure $\square^{*}$ ). This proof is quite straightforward, whereas the generalisation to $\mathrm{PDL}_{\omega}$ is rather involved. As far as we know, the disharmony results are new.

In Kooi (2003) and Renardel de Lavalette et al. (2002), earlier versions of this paper, the proofs are more involved: the identity of saturated and maximal consistent sets was not discovered yet, and a different countermodel for modal harmony was used.

\section{The Infinitary Proof System PDL $\omega$}

The infinitary proof system $\mathrm{PDL}_{\omega}$ is an extension of the usual axiom system for PDL, with respect to the same language and the same Kripke semantics. As a reminder, we repeat the definitions of both language and semantics (for more information on PDL, see Harel et al. (2000)). Then we present our proof system for PDL ${ }_{\omega}$.

Definition 1 (Language of $P D L$ ) Let a countable set of propositional variables $P$ and a countable set of atomic programs $\Pi$ be given. The language $\mathcal{L}=\mathcal{L}_{P \Pi}$ of PDL consists of a set of formulas $\varphi$ and the set of programs $\alpha$, given by the following rules (where $p \in P, a \in \Pi$ ):

$$
\begin{aligned}
& \varphi::=\perp|p| \neg \varphi|(\varphi \rightarrow \psi)|[\alpha] \varphi \\
& \alpha::=a|\alpha ; \beta| \alpha \cup \beta\left|\alpha^{*}\right| ? \varphi
\end{aligned}
$$

Definition 2 (Models of $P D L$ ) A model for $\mathcal{L}$ is a tuple $M=(W, R, V)$ such that:

$$
\begin{array}{ll}
W \neq \emptyset & \text { (a set of states or possible worlds) } \\
R(a) & \text { (a binary relation on } W \text { for each a in aprograms) } \\
V: P \rightarrow \wp(W) & \text { (assigns } \text { a set of states to each } p \in P)
\end{array}
$$

A pair $F=(W, R)$ is called a frame.

The truth definition is as expected for normal modal logics. As a reminder, here follows the modal clause:

$$
(M, w) \models[\alpha] \varphi \text { iff }(M, v) \models \varphi \text { for all } v \text { with } w R(\alpha) v
$$

where $R$ is extended to arbitrary programs in the following way: 


$$
\begin{aligned}
& R(\alpha ; \beta)=R(\alpha) \circ R(\beta) \\
& R(\alpha \cup \beta)=R(\alpha) \cup R(\beta) \\
& R\left(\alpha^{*}\right)=R(\alpha)^{*} \\
& R(? \varphi) \quad=\{(w, w) \mid(M, w) \models \varphi\}
\end{aligned}
$$

Here $R(\alpha)^{*}$ denotes the reflexive transitive closure of $R(\alpha)$.

By $\Gamma \models \varphi$ we mean the local consequence relation, i.e. $\Gamma \models \varphi$ iff for every model $M$ and world $w,(M, w) \models \psi$ for every $\psi \in \Gamma$ implies that $(M, w) \models \varphi$.

Definition 3 (Axioms for PDL)

Taut all instantiations of propositional tautologies

$$
\begin{aligned}
& \operatorname{Distr}[\alpha](\varphi \rightarrow \psi) \rightarrow([\alpha] \varphi \rightarrow[\alpha] \psi) \\
& \operatorname{PAX}[? \varphi] \psi \leftrightarrow(\varphi \rightarrow \psi) \\
& ; \operatorname{AX}[\alpha ; \beta] \varphi \leftrightarrow[\alpha][\beta] \varphi \\
& \cup \operatorname{AX}[\alpha \cup \beta] \varphi \leftrightarrow([\alpha] \varphi \wedge[\beta] \varphi) \\
& * \operatorname{AX}\left[\alpha^{*}\right] \varphi \leftrightarrow\left(\varphi \wedge[\alpha]\left[\alpha^{*}\right] \varphi\right)
\end{aligned}
$$

Observe that we omitted the induction axiom for $\alpha^{*}$ : see the remark after Definition 4.

We now extend the system PDL to an infinitary proof system PDL ${ }_{\omega}$ by inductively defining a derivation relation $\Gamma \vdash \varphi$ ( $\varphi$ a formula, $\Gamma$ a set of formulas). Notice that in the following definition, the language remains finitary (all formulas have finite length) and only the rule Inf* is non-standard. Besides the usual shorthand notation $\Gamma, \varphi$ for $\Gamma \cup\{\varphi\}, \Gamma, \Delta$ for $\Gamma \cup \Delta, \vdash \varphi$ for $\emptyset \vdash \varphi$, and $\varphi_{1}, \ldots, \varphi_{n} \vdash \psi$ for $\left\{\varphi_{1}, \ldots, \varphi_{n}\right\} \vdash \psi$, we shall also write:

$$
\begin{array}{ll}
\Gamma \vdash \Delta & \text { for } \Gamma \vdash \varphi \text { for all } \varphi \in \Delta \\
{[\alpha] \Gamma} & \text { for }\{[\alpha] \varphi \mid \varphi \in \Gamma\} \\
{[\alpha]^{-1} \Gamma \text { for }\{\varphi \mid[\alpha] \varphi \in \Gamma\}}
\end{array}
$$

Definition 4 (Infinitary derivation relation for $\mathrm{PDL}_{\omega}$ ) $\Gamma \vdash \varphi$ is defined as the smallest relation closed under the following rules:

$$
\begin{array}{lll}
\text { AX } & \vdash \varphi \text { if } \varphi \text { is an axiom of } \mathrm{PDL} & \\
\mathrm{MP} \quad \varphi, \varphi \rightarrow \psi \vdash \psi & \text { (modus ponens) } \\
\text { Inf* }^{*}\left\{\left[\alpha ; \beta^{n}\right] \varphi \mid n \in \mathbb{N}\right\} \vdash\left[\alpha ; \beta^{*}\right] \varphi & \text { (infinitary *-introduction) } \\
\text { SNec if } \Gamma \vdash \varphi \text { then }[\alpha] \Gamma \vdash[\alpha] \varphi & \text { (strong necessitation) } \\
\text { Ded if } \Gamma, \varphi \vdash \psi \text { then } \Gamma \vdash \varphi \rightarrow \psi & \text { (deduction) } \\
\text { W } \quad \text { if } \Gamma \vdash \varphi \text { then } \Gamma, \Delta \vdash \varphi & \text { (weakening) } \\
\text { Cut if } \Gamma \vdash \Delta \text { and } \Gamma, \Delta \vdash \varphi \text { then } \Gamma \vdash \varphi &
\end{array}
$$


The choice of this particular collection of rules is mainly based on what is needed to make the completeness theorem go through. We do not exclude the possibility that the collection is not minimal. It might be possible that, e.g., the infinitary Cut rule is a derived rule, but we think the proof will not be straightforward, and will require additional rules, e.g. the inverse of the Deduction rule.

The rule Inf* is given in terms of modalities of the form $[\alpha ; \beta]$. This will turn out to be useful later, when we prove Lemma 1 on saturated sets. We can, for instance, apply the rule to formulas of the form $\varphi \rightarrow[\alpha] \psi$ by converting them to formulas of the form $[? \varphi ; \alpha] \psi$.

It is an easy exercise to show that the induction axiom

$$
\varphi \wedge\left[\alpha^{*}\right](\varphi \rightarrow[\alpha] \varphi) \rightarrow\left[\alpha^{*}\right] \varphi
$$

is derivable via Inf* and $* \mathrm{AX}$.

It is not hard to verify that the rules of $\mathrm{PDL}_{\omega}$ are sound with respect to the semantics of PDL (i.e. $\Gamma \vdash \varphi$ implies that $\Gamma \models \varphi$ ). We shall show in Sect. 3 that $\mathrm{PDL}_{\omega}$ is also strongly complete with respect to these semantics.

\section{Strong Completeness: The Canonical Model for $\mathrm{PDL}_{\omega}$}

In this section, we prove strong completeness of $\mathrm{PDL}_{\omega}$. But first we explain where the problem lies.

\subsection{The Problem in Proving Strong Completeness}

The obvious idea would be to apply the Henkin construction of a canonical model from maximal consistent sets of formulas, and to prove Lindenbaum's Lemma for PDL $\omega$ that every consistent set can be extended to a maximal consistent set. The last part is problematic: the limit construction in Lindenbaum's lemma yields a set which is maximal, closed under Inf* and only finitely $\vdash$-closed. We call this type of sets saturated sets (Definition 5). Now the problem is that saturated sets $\Gamma$ may be inconsistent, in the sense that $\Gamma \vdash \perp$ is not excluded by the definition of saturatedness, which only entails that $\Gamma^{\prime} \forall \perp$ for finite $\Gamma^{\prime} \subseteq \Gamma$. (If $\vdash$ were compact, then all saturated sets evidently were maximal consistent, but that is not the case for $\mathrm{PDL}_{\omega}$.) However, we are able to show that all saturated sets are maximal consistent (Lemma 1). The proof goes by showing that saturated sets are fully (and not only finitely) $\vdash$-closed. Although this notion of saturated sets has been used by e.g. Goldblatt in (1993) and Segerberg in (1994) in the context of strong completeness for infinitary modal logics, the fact that they coincide with maximal consistent sets has not been observed before, as far as we know. This leads to a rather short argument for strong completeness in comparison with Goldblatt (1993) and Segerberg (1994). 


\subsection{How to Prove Strong Completeness}

Definition 5 (Saturated sets) A collection of formulas $\Gamma$ is called saturated if:

- $\quad \Gamma$ is finitely $\vdash$-closed: if $\Gamma^{\prime} \subseteq \Gamma$ is finite and $\Gamma^{\prime} \vdash \varphi$, then $\varphi \in \Gamma$;

- $\quad \Gamma$ is Inf*-closed: if $\left\{\left[\alpha ; \beta^{n}\right] \varphi \mid n \in \mathbb{N}\right\} \subseteq \Gamma$, then $\left[\alpha ; \beta^{*}\right] \varphi \in \Gamma$;

- $\quad \Gamma$ is maximal: $\varphi \notin \Gamma \Leftrightarrow \neg \varphi \in \Gamma$ for every formula $\varphi$.

It is quite easy to show that any saturated set $\Gamma$ contains all derivable formulas, does not contain $\perp$ and satisfies $(\varphi \rightarrow \psi) \in \Gamma \Leftrightarrow(\varphi \in \Gamma \Rightarrow \psi \in \Gamma)$. We shall use these properties of saturated sets freely below without reference. Furthermore we recall the obvious fact that a collection of formulas $\Gamma$ is maximal consistent iff it is consistent (i.e. $\Gamma \nvdash \perp$ ) and $\Gamma$ contains exactly one from $\varphi, \neg \varphi$ for every formula $\varphi$ in the language $\mathcal{L}$. Now we prove that the concepts of saturated and maximal consistent sets coincide.

Lemma 1 (Saturation lemma for $\mathrm{PDL}_{\omega}$ ) A set is saturated if and only if it is maximal consistent.

Proof The part from right to left is simple, and follows directly from the definition. For the other direction, let $\Gamma$ be saturated. Maximality follows directly from the third clause of Definition 5 of saturated set, so we only have to show consistency. For this, it suffices to show that $\Gamma$ contains all its consequences, i.e.

$$
\Gamma \vdash \varphi \text { implies } \varphi \in \Gamma \text {. }
$$

For, from (1) and the fact that $\perp \notin \Gamma$, it follows that $\Gamma \nvdash \perp$. We prove a more general statement than (1), namely: for all $\alpha, \varphi$ and $\Delta$,

$$
\text { if } \Delta \vdash \varphi \text { and }[\alpha] \Delta \subseteq \Gamma \text {, then }[\alpha] \varphi \in \Gamma \text {. }
$$

Before we prove this statement, we show how (2) implies (1), using the fact that for all $\psi$ we have by ?AX that $\vdash \psi \leftrightarrow[\top$ ?] $\psi$, hence $\psi \in \Gamma \Leftrightarrow[\top$ ?] $\psi \in \Gamma$. Now, suppose the antecedent of (1) holds, i.e. $\Gamma \vdash \varphi$, then $\Gamma \vdash[\top$ ?] $\varphi$; also [ $\top$ ?] $\Gamma \subseteq \Gamma$ (since $\Gamma$ is finitely $\vdash$-closed). By (2), we obtain [ $\top$ ?] $\varphi \in \Gamma$, so $\varphi \in \Gamma$.

Now we prove (2) with induction over a derivation of $\Delta \vdash \varphi$. So we assume $\Delta \vdash \varphi$ and $[\alpha] \Delta \subseteq \Gamma$, we want to show $[\alpha] \varphi \in \Gamma$, and we consider the last rule applied in the derivation of $\Delta \vdash \varphi$ :

- AX: so $\Delta=\emptyset$ and $\varphi$ is an axiom of PDL. Now $\vdash[\alpha] \varphi$ (by SNec), and therefore $[a] \varphi \in \Gamma$, since $\Gamma$ is saturated.

- MP: so $\Delta=\{\psi, \psi \rightarrow \varphi\}$ for some $\psi$. Now $[\alpha] \psi,[\alpha](\psi \rightarrow \varphi) \in \Gamma$. Since $[\alpha](\psi \rightarrow \varphi) \vdash([\alpha] \psi \rightarrow[\alpha] \varphi)$, also by saturation $([\alpha] \psi \rightarrow[\alpha] \varphi) \in \Gamma$, so $[\alpha] \varphi \in \Gamma$.

- Inf*: so $\Delta=\left\{\left[\beta ; \gamma^{n}\right] \psi \mid n \in \mathbb{N}\right\}$ and $\varphi=\left[\beta ; \gamma^{*}\right] \psi$ for some $\beta, \gamma, \psi$. We have $\left\{[\alpha]\left[\beta ; \gamma^{n}\right] \psi \mid n \in \mathbb{N}\right\} \subseteq \Gamma$, then $\left\{\left[(\alpha ; \beta) ; \gamma^{n}\right] \psi \mid n \in \mathbb{N}\right\} \subseteq \Gamma$, so $\left[(\alpha ; \beta) ; \gamma^{*}\right] \psi \in \Gamma$ (for $\Gamma$ is closed w.r.t. Inf*), hence $[\alpha] \varphi=[\alpha]\left[\beta ; \gamma^{*}\right] \psi \in \Gamma$. 
- SNec: so $\Delta=[\beta] \Theta$ and $\varphi=[\beta] \chi$ for some $\beta, \Theta, \chi$, and $\Theta \vdash \chi$. By the induction hypothesis, we have $[\alpha ; \beta] \Theta \subseteq \Gamma \Rightarrow[\alpha ; \beta] \chi \in \Gamma$. Now $[\alpha] \varphi \in \Gamma$ follows from $[\alpha][\beta] \Theta \subseteq \Gamma \Leftrightarrow[\alpha ; \beta] \Theta \subseteq \Gamma$ and $[\alpha ; \beta] \chi \in \Gamma \Leftrightarrow[\alpha][\beta] \chi \in \Gamma$.

- Ded: so $\varphi=\psi \rightarrow \chi$ for some $\psi, \chi$, and $\Delta, \psi \vdash \chi$. By the induction hypothesis, we have $[\alpha ; \psi$ ? ] $\Delta \cup\{[\alpha ; \psi$ ? ] $\psi\} \subseteq \Gamma \Rightarrow[\alpha ; \psi$ ?] $\chi \in \Gamma$. First we observe $\vdash[\alpha ; \psi$ ?] $\psi$, so $[\alpha ; \psi$ ?] $\psi \in \Gamma$. Now assume $[\alpha] \Delta \subseteq \Gamma$, then $[\alpha ; \psi$ ?] $\Delta \subseteq \Gamma$ (for $[\alpha] \gamma \vdash[\alpha ; \psi$ ?] $\gamma$ for all formulas $\gamma)$, so $[\alpha ; \psi$ ?] $\chi \in \Gamma$, hence $[\alpha](\psi \rightarrow \chi) \in \Gamma$ (for $\vdash[\alpha ; \psi ?] \chi \leftrightarrow[\alpha](\psi \rightarrow \chi)$ ), i.e. $[\alpha] \varphi \in \Gamma$.

- W: direct consequence of the induction hypothesis.

- Cut: so for some $\Theta$ we have $\Delta \vdash \theta$ for all $\theta \in \Theta$, and $\Delta, \Theta \vdash \varphi$. By the induction hypothesis, $[\alpha] \theta \in \Gamma$ for all $\theta \in \Theta$, i.e. $[\alpha] \Theta \subseteq \Gamma$, hence $[\alpha](\Delta \cup \Theta) \subseteq \Gamma$. Now apply the induction hypothesis to $\Delta, \Theta \vdash \varphi$, and we obtain $[\alpha] \varphi \in \Gamma$.

Lemma 2 (Lindenbaum lemma for $\mathrm{PDL}_{\omega}$ ) Every consistent set can be extended to a maximal consistent set.

Proof By Lemma 1, it suffices to show that it is possible to extend a given consistent set to a saturated one. Let $\Delta$ be a consistent set, i.e. $\Delta \forall \forall \perp$. Let $\left\{\varphi_{n} \mid n \in \mathbb{N}\right\}$ be an enumeration of all PDL-formulas. We shall inductively define an increasing sequence $\left\{\Gamma_{n} \mid n \in \mathbb{N}\right\}$ of formula sets extending $\Delta$, and show that $\Gamma=\bigcup\left\{\Gamma_{n} \mid n \in \mathbb{N}\right\}$ is saturated.

$$
\begin{aligned}
& \Gamma_{0}=\Delta \\
& \Gamma_{n+1}= \begin{cases}\Gamma_{n} \cup\left\{\varphi_{n}\right\} & \text { if } \Gamma_{n} \vdash \varphi_{n} \\
\Gamma_{n} \cup\left\{\neg \varphi_{n}\right\} & \text { if } \Gamma_{n} \nvdash \varphi_{n} \text { and } \varphi_{n} \text { is not } \\
& \text { of the form }\left[\alpha ; \beta^{*}\right] \psi \\
\Gamma_{n} \cup\left\{\neg \varphi_{n}, \neg\left[\alpha ; \beta^{k}\right] \psi\right\} & \text { otherwise, where } k \text { is the } \\
& \text { least natural number } \\
& \text { such that } \Gamma_{n} \nvdash\left[\alpha ; \beta^{k}\right] \psi \\
& \text { (and } \varphi_{n} \text { is of the form } \\
& \left.\left[\alpha ; \beta^{*}\right] \psi\right)\end{cases}
\end{aligned}
$$

We observe that the $k$ in the last case always exists: for if $\Gamma_{n} \vdash\left[\alpha ; \beta^{k}\right] \psi$ for all $k \in \mathbb{N}$, then (by Inf* and Cut) $\Gamma_{n} \vdash\left[\alpha ; \beta^{*}\right] \psi$, contradicting $\Gamma_{n} \not \forall \varphi_{n}$. So the definition of $\Gamma_{n}$ is correct.

All $\Gamma_{n}$ are consistent: this is shown with induction over $n$, using the consistency of $\Delta$ for the base case. The induction step is easy when the first or second case in the definition of $\Gamma_{n+1}$ applies. If the last case applies and $\Gamma_{n+1}$ were inconsistent, then $\Gamma_{n} \vdash\left[\alpha ; \beta^{*}\right] \psi \vee\left[\alpha ; \beta^{k}\right] \psi$ via Ded, so, by using $* \operatorname{AX} k$ times, $\Gamma_{n} \vdash\left[\alpha ; \beta^{k}\right] \psi$, contradicting the definition of $k$.

We claim that $\Gamma$ is saturated. Maximality and closure with respect to Inf* follow directly from the definition of the $\Gamma_{n}$ (where the third case ensures that Inf* holds). For finite $\vdash$-closure we argue via contradiction: let $\Gamma^{\prime} \subseteq \Gamma$ be finite with $\Gamma^{\prime} \vdash \varphi$ and assume that $\varphi \notin \Gamma$ : then (by maximality) $\neg \varphi \in \Gamma$, so $\Gamma^{\prime} \cup\{\neg \varphi\} \subseteq \Gamma$, hence $\Gamma^{\prime} \cup\{\neg \varphi\} \subseteq \Gamma_{n}$ for some $n$; but $\Gamma_{n}$ is consistent and $\Gamma^{\prime} \cup\{\neg \varphi\} \vdash \perp$, and we have a contradiction. Finally, because $\Gamma$ is saturated, it is maximal consistent by Lemma 1 .

Now we can define the canonical model. 
Definition 6 (Canonicalmodel) We define the canonical Kripke model $M=(W, R, V)$ by

$$
\begin{aligned}
& W=\{\Gamma \mid \Gamma \text { maximal consistent }\} \\
& R(a)=\left\{(\Gamma, \Delta) \in W^{2} \mid[a]^{-1} \Gamma \subseteq \Delta\right\} \\
& V(p)=\{\Gamma \in W \mid p \in \Gamma\}
\end{aligned}
$$

The Truth lemma shows that $(M, \Gamma) \models p \Leftrightarrow p \in \Gamma$ extends to all formulas of the language:

Lemma 3 (Truth lemma) For all $\Gamma \in W$ and for all formulas $\varphi \in \mathcal{L}$, we have $(M, \Gamma) \models \varphi$ iff $\varphi \in \Gamma$.

Proof Induction over $\varphi$. The atomic and propositional cases are standard. We will prove the case $\varphi=[\alpha] \psi$, by induction over $\alpha$; the cases for complex programs $\alpha$ of the forms ? $\chi, \beta ; \gamma$ and $\beta \cup \gamma$ are easy (using the corresponding axioms), so we only give the proofs of the remaining two cases $\alpha=a$ and $\alpha=\beta^{*}$. Note that the proof as a whole has the form of an induction over a well-ordering of formulas, where $\left[\alpha^{n}\right] \varphi$ is considered to be a subformula of $\left[\alpha^{*}\right] \varphi$.

1. $\alpha=a$, atomic. Using the definition of the truth relation and the induction hypothesis $(M, \Delta) \models \psi \Leftrightarrow \psi \in \Delta$ for all $\Delta \in W$, we see that $(M, \Gamma) \models[a] \psi$ is equivalent to

$$
\text { for all } \Delta \in W(\Gamma R(a) \Delta \Rightarrow \psi \in \Delta)
$$

It is evident that (3) follows from $[a] \psi \in \Gamma$. To see that (3) implies $[a] \psi \in \Gamma$ as well, we argue via contraposition. So assume $[a] \psi \notin \Gamma$, i.e. (by maximal consistency) $\neg[a] \psi \in \Gamma$. We shall show that there is a maximal consistent $\Delta$ with $\theta \in \Delta$ for all $\theta$ such that $[a] \theta \in \Gamma$, and $\neg \psi \in \Delta$. By the Lindenbaum lemma, it suffices to show that $\{\chi \mid[a] \chi \in \Gamma\} \cup\{\neg \psi\}$ is consistent. Assume it is not, i.e. $\{\chi \mid[a] \chi \in \Gamma\} \cup\{\neg \psi\} \vdash \perp$, then $\{\chi \mid[a] \chi \in \Gamma\} \vdash \psi$ via Ded. Thus, with SNec: $\{[a] \chi \mid[a] \chi \in \Gamma\} \vdash[a] \psi$. Hence a fortiori $\Gamma \vdash[a] \psi$ and $[a] \psi \in \Gamma$, contradicting the assumption $[a] \psi \notin \Gamma$. Therefore $\Delta$ is consistent, and for all $\chi([a] \chi \in \Gamma \Rightarrow \chi \in \Delta)$, however $\psi \notin \Delta$. Therefore (3) is not the case.

2. $\alpha=\beta^{*}:(M, \Gamma) \models\left[\beta^{*}\right] \psi \Leftrightarrow$ for all $n \in \mathbb{N}(M, \Gamma) \models\left[\beta^{n}\right] \psi \Leftrightarrow\left(\left[\beta^{n}\right] \psi \in \Gamma\right.$ for all $n \in \mathbb{N}) \Leftrightarrow\left[\beta^{*}\right] \psi \in \Gamma$, using the induction hypothesis in the second step, and $* A X$, Inf* in the last step.

Note that in the Truth lemma, we do not prove the dual property for programs, namely, $\Gamma R(\alpha) \Delta$ iff $\varphi \in \Delta$ for all $\varphi$ such that $[\alpha] \varphi \in \Gamma$ (it holds by definition for atomic programs $a$ ). In Sect. 4 we elaborate on this lack of "full harmony" (Definition 7).

Theorem 1 (Strong completeness of $\mathrm{PDL}_{\omega}$ ) Let $S$ be the class of all Kripke frames for the language $\mathcal{L}$. Then for all formulas $\varphi$ and all sets of formulas $\Phi, \Phi \models_{S} \varphi$ implies $\Phi \vdash \varphi$. 
Proof By contraposition. Suppose $\Phi \nvdash \varphi$, then $\Phi \cup\{\neg \varphi\}$ is consistent. By Lemma 2, $\Phi \cup\{\neg \varphi\}$ is extended to a maximal consistent set $\Gamma$ with $\neg \varphi \in \Gamma$ and $\Phi \subseteq \Gamma$. Now by Lemma 3, we conclude that in the canonical Kripke model, $(M, \Gamma) \not \models \varphi$ and $(M, \Gamma) \models \Phi$, as desired.

Note that the completeness proof immediately gives a canonical standard model, contrary to the early proofs of weak completeness for PDL as they appear in Kozen and Parikh (1981) and Harel et al. (2000), which use nonstandard models.

\subsection{Adapting the Proof Method to Other Modal Logics}

In this subsection, we indicate how to generalize the strong completeness proof for $\mathrm{PDL}_{\omega}$ to other modal logics with infinitary axiomatizations. Let $\mathrm{ML}_{\omega}$ be an arbitrary modal logic, based on a countable language with modalities $\{[\pi] \mid \pi \in \Pi\}$, and a derivability relation $\vdash$ generated by the axiom and rule schemata Taut, Distr, MP, SNec, Ded, W, Cut (see Definitions 3 and 4) and the countable set of rules Rules $=\left\{\left(\Gamma_{n}, \varphi_{n}\right) \mid n \in \mathbb{N}\right\}$. We discuss examples of $\mathrm{ML}_{\omega}$ at the end of this subsection. Let Cond (conditionalization) be the proof rule if $\Gamma \vdash \varphi$, then $(\psi \rightarrow \Gamma) \vdash \psi \rightarrow \varphi$, where $\psi \rightarrow \Gamma$ abbreviates $\{\psi \rightarrow \gamma \mid \gamma \in \Gamma\}$. We leave it as an exercise to the reader to show that Cond is a derived rule both in $\mathrm{PDL}_{\omega}$ and in $\mathrm{ML}_{\omega}$ (hint: use MP, W, Cut, Ded). Without loss of generality, we may assume that Rules is closed under SNec and Cond, for closing off a countable set of rules under SNec and Cond can be done in countably many steps, so the resulting set of rules is still countable.

In the language of PDL, implications $\psi \rightarrow \varphi$ can be written (modulo equivalence) as modal formulas $[\psi ?] \varphi$. This is in general not possible in $\mathrm{ML}_{\omega}$, and therefore we introduce pseudo-modalities: finite (possibly empty) sequences $s=\left(s_{1}, \ldots, s_{n}\right)$ of modalities $\pi \in \Pi$ and formulas $\psi$. [s] $[\varphi$ abbreviates a formula, defined as follows:

$$
\begin{aligned}
& {[()] \varphi \quad=\varphi} \\
& {\left[\left(\psi, s_{2}, \ldots, s_{n}\right)\right] \varphi=\psi \rightarrow\left[\left(s_{2}, \ldots, s_{n}\right)\right] \varphi} \\
& {\left[\left(\pi, s_{2}, \ldots, s_{n}\right)\right] \varphi=[\pi]\left[\left(s_{2}, \ldots, s_{n}\right)\right] \varphi}
\end{aligned}
$$

The idea is that, e.g., $\left[\left(\pi_{1}, \psi, \pi_{2}\right)\right] \varphi$ is an abbreviation of the formula $\left[\pi_{1}\right](\psi \rightarrow$ $\left[\pi_{2}\right] \varphi$ ). With induction over the length of $s$, we can prove that the following generalizations of Distr and SNec to pseudo-modalities hold:

$$
\begin{aligned}
& \vdash[s](\varphi \rightarrow \psi) \rightarrow([s] \varphi \rightarrow[s] \psi) \\
& \text { if } \Gamma \vdash \varphi \text { then }[s] \Gamma \vdash[s] \varphi
\end{aligned}
$$

In the definition of saturated sets, we now require closure under Rules (instead of Inf*). The proof that saturated sets in $\mathrm{ML}_{\omega}$ are maximal consistent follows the lines of the proof for Lemma 1 with, instead of (2), the induction hypothesis

$$
\text { if } \Delta \vdash \varphi \text { and }[s] \Delta \subseteq \Gamma \text {, then }[s] \varphi \in \Gamma
$$

for all pseudo-modalities $s$. The induction step for the case that $\Delta \vdash \varphi$ is an instance of Rules is easy, using that Rules is closed under SNec and Cond. 
In the limit construction in the proof of the Lindenbaum lemma, we replace Inf* by Rules in the definition of $\Delta_{n+1}$. In the case that (unlike Inf*) Rules has instances with identical conclusions, some care must be taken to treat all instances properly.

The canonical model for $\mathrm{ML}_{\omega}$ is defined as for $\mathrm{PDL}_{\omega}$ in Definition 6, with one adaptation: for $\mathrm{PDL}_{\omega}$, the accessibility relation is defined only for atomic modalities, while here we define $R(\pi)$ for all modalities $\pi \in \Pi$. The proof of the Truth lemma for the canonical model of $\mathrm{ML}_{\omega}$ runs parallel to the proof of Lemma 3: the last step for the case $\alpha=\beta^{*}$ is not needed here. Now soundness and strong completeness of $\mathrm{ML}_{\omega}$ with respect to models (not frames) satisfying Rules follows straightforwardly.

Note that we do not automatically obtain strong completeness with respect to the class of frames, since we cannot guarantee in general that the frame of the canonical model satisfies Rules. Thus, $\mathrm{ML}_{\omega}$ need not be canonical, in contrast to $\mathrm{PDL}_{\omega}$ and the examples treated in the next subsection.

\subsubsection{Some Examples}

We shortly present some examples of infinitary modal logics for which we can prove strong completeness. The first two are taken from Segerberg (1994). BCC is the modal logic that characterizes frames $(W, R)$ that satisfy the bounded chain condition $\forall w \in W \exists n \forall v \in W \forall m\left((w, v) \in R^{m} \rightarrow m<n\right)$, i.e. the length of paths starting in some world is bounded. BCC is axiomatized by the usual axioms for the minimal modal logic $\mathrm{K}$ and the infinitary rule $\left\{\nabla^{n} \top \mid n \in \mathbb{N}\right\} \vdash \perp$. So BCC is an instance of $\mathrm{ML}_{\omega}$ where Rules has one element; moreover, the frame of the canonical model satisfies BCC so it is canonical.

Another example is ancestral logic with two modalities $\square$ and $\square^{*}$, axiomatized by $\left\{\square^{n} \varphi \mid n \in \mathbb{N}\right\} \vdash \square^{*} \varphi$. The accessibility relation $R\left(\square^{*}\right)$ of $\square^{*}$ is the reflexive transitive closure of $R(\square)$. Like PDL ${ }_{\omega}$, this is not exactly an instance of $\mathrm{ML}_{\omega}$, since there is additional structure between the modalities. As a consequence, the definition of the canonical model only contains a clause for $R(\square)$, since the definition of $R\left(\square^{*}\right)$ is part of the definition of model of ancestral logic. Again, the frame of the canonical model satisfies ancestral logic, so it is canonical.

A third example (in fact a bundle of examples) is epistemic logic with a common knowledge operator, where we have a collection $\mathcal{A}$ of agents, and modalities $\square_{a}$ (with the intended meaning ' $a$ knows that ..., for each agent $a \in \mathcal{A}$ ), $E$ (general knowledge 'everybody knows that ...') and $C$ (common knowledge), axiomatized by

$$
\begin{aligned}
& \vdash E \varphi \leftrightarrow \bigwedge_{a \in \mathcal{A}} \square_{a} \varphi \\
& \vdash C \varphi \leftrightarrow E(\varphi \wedge C \varphi) \\
& \left\{[s] E^{n+1} \varphi \mid n \in \mathbb{N}\right\} \vdash[s] C \varphi
\end{aligned}
$$

where $s$ ranges over the pseudo-modalities. Several variants can be obtained by adding one or more of the usual axioms for epistemic logic, viz. $\square_{a} \varphi \rightarrow \varphi, \square_{a} \varphi \rightarrow \square_{a} \square_{a} \varphi$ and $\neg \square_{a} \varphi \rightarrow \square_{a} \neg \square_{a} \varphi$. In the semantics, the accessibility relation $R(E)$ of $E$ is the union of the accessibility relations $R\left(\square_{a}\right)$ for all $a \in \mathcal{A}$, and $R(C)$ is the transitive closure of $R(E)$. The definition of the canonical model for each of the variants 
only contains clauses for $R\left(\square_{a}\right)$ for $a \in \mathcal{A}$. All variants are strongly complete and canonical with respect to their semantics.

In Kooi et al. (2006), we investigate hybrid versions of infinitary modal logics. The presence of nominals enhances the expressive power, and we show there that several hybrid logics characterizing certain frame conditions have a straightforward axiomatization: e.g. ancestral logic, reachability logic (where every two worlds are connected via an $R$-path), cycle logic (where every world is contained in some cycle) and bounded chain logic.

\section{Modal (dis)harmony}

In this section we take a closer look at the canonical model defined in Sect. 3. The construction of a canonical model from maximally consistent sets is the standard technique in completeness proofs: maximal consistent sets provide the bridge between syntax and semantics that facilitates the completeness theorem. Therefore one would expect the following property for the canonical model.

Definition 7 (Full harmony) A canonical model $M$ is fully harmonious for a logic containing formulas $\varphi$ and modalities $[\alpha]$ iff for all maximal consistent sets $\Gamma$ and $\Delta$, and for all $\varphi$ and all modalities $[\alpha]$ :

$$
\begin{aligned}
& \text { formula harmony: }(M, \Gamma) \models \varphi \text { iff } \varphi \in \Gamma \text { and } \\
& \text { modal harmony: }(\Gamma, \Delta) \in R(\alpha) \text { iff }[\alpha]^{-1} \Gamma \subseteq \Delta
\end{aligned}
$$

where $R(\alpha)$ is the accessibility relation associated with the modality $[\alpha]$, and $[\alpha]^{-1} \Gamma$ abbreviates $\{\varphi \mid[\alpha] \varphi \in \Gamma\}$.

Formula harmony generalizes a property of atomic formulas that holds in the canonical model by definition, and modal harmony does the same with atomic modalities (such as atomic programs or individual epistemic operators). In the Truth Lemma (Lemma 3), we proved that the canonical model for $\mathrm{PDL}_{\omega}$ has formula harmony. Modal harmony was only needed there for atomic modalities: the semantic properties of the accessibility relations for composite modalities are sufficient to prove the Truth Lemma.

Only when focusing on modal harmony as an interesting property in itself, one notices that it does not hold for the canonical model. In Kozen and Parikh (1981) modal disharmony (i.e. failure of modal harmony) was claimed without proof for finite canonical models of PDL. We found it quite surprising that the infinite canonical model for $\mathrm{PDL}_{\omega}$ also lacks modal harmony. It does hold from left to right, however:

$$
\text { if }(\Gamma, \Delta) \in R(\alpha) \text { then }[\alpha]^{-1} \Gamma \subseteq \Delta
$$

There is an easy proof for this statement that uses formula harmony, i.e. the Truth Lemma. The converse implication (if $[\alpha]^{-1} \Gamma \subseteq \Delta$ then $(\Gamma, \Delta) \in R(\alpha)$ ) holds for tests and is preserved under sequential composition and non-deterministic choice, but not under iteration. The remainder of this section is devoted to showing that modal 
harmony fails. We first prove this for ancestral logic in Sect. 4.1, as a stepping-stone towards proving the more difficult case for $\mathrm{PDL}_{\omega}$ in Sect. 4.2.

\subsection{The Case for Ancestral Logic}

Ancestral logic is introduced in Subsect. 3.3.1. In this section we take the language for ancestral logic $\mathcal{L}\left(\square^{*}\right)$ with an empty set of propositional variables.

In order to show that modal harmony fails, we have to find two maximal consistent sets $\Gamma, \Delta$ with $\left(\square^{*}\right)^{-1} \Gamma \subseteq \Delta$ while $(\Gamma, \Delta) \notin R^{*}$; here the relation $R=R(\square)$ on maximal consistent sets is defined by

$$
R=\left\{\left(\Gamma_{1}, \Gamma_{2}\right) \mid \Gamma_{1}, \Gamma_{2} \text { maximal consistent and } \square^{-1} \Gamma_{1} \subseteq \Gamma_{2}\right\}
$$

To find $\Gamma$ and $\Delta$, we use the fact that the collection of formulas that hold in a world in a model is maximal consistent. So it suffices to come up with a countermodel for modal harmony, i.e. a model $M=(W, R, V)$ with $\gamma, \delta \in W$ such that $\Gamma=\{\varphi \mid M, \gamma \models \varphi\}$ and $\Delta=\{\varphi \mid M, \delta \models \varphi\}$ satisfy $\left(\square^{*}\right)^{-1} \Gamma \subseteq \Delta$ and $(\Gamma, \Delta) \notin R^{*}$. We provide such a countermodel, where $\omega$ plays the role of $\delta$.

Definition 8 (Countermodel) Let $M_{c}=\left(\mathbb{Z} \cup\{\gamma, \omega\}, R_{c}, V_{c}\right)$ be a model for ancestral logic, where

$$
\begin{aligned}
R_{c}= & \{(x, y) \in \mathbb{N} \times \mathbb{N} \mid x>y\} \cup \\
& \{(-x, x),(-x,-x-1) \mid x \in[1, \omega)\} \cup \\
& \{(\omega, x) \mid x \in \mathbb{N}\} \cup\{(\gamma,-1)\}
\end{aligned}
$$

and $V_{c}=\varnothing$.

See Fig. 1 for a picture of this model. The idea is that modal harmony fails with respect to the maximal consistent sets $\Gamma=\{\varphi \mid M, \gamma \models \varphi\}$ and $\Delta=\{\varphi \mid M, \omega \models \varphi\}$ for the modality $\square^{*}$. The proof depends on two lemmas. The first lemma (Lemma 4) shows that $(\Gamma, \Delta) \notin R^{*}$. (From the picture of the model it is quite clear that $(\gamma, \omega) \notin R_{c}^{*}$, but we have to prove the corresponding property for the maximal consistent sets associated with these worlds.) The second lemma (Lemma 5) states that a formula is true in $\omega$ iff its extension is cofinite. This will be used to show that $\gamma \models \square^{*} \varphi$ implies that $\omega \models \varphi$, because if $\gamma \models \square^{*} \varphi$, then the extension of $\varphi$ is cofinite.

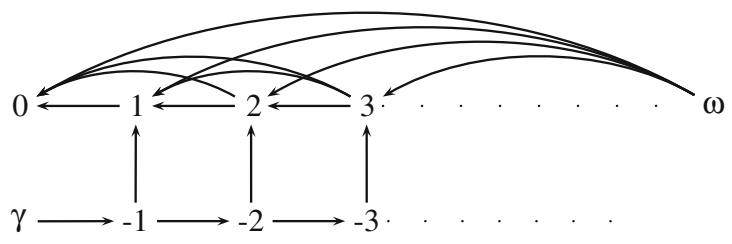

Fig. 1 The countermodel $M$ 
Lemma 4 It is not the case that $(\Gamma, \Delta) \in R^{*}$.

Proof If it were the case that $(\Gamma, \Delta) \in R^{*}$, then there would be an $n$ such that $(\Gamma, \Delta) \in R^{n}$. As was noted above, modal harmony does hold from left to right, so that would imply that $\left(\square^{n}\right)^{-1} \Gamma=\left\{\varphi \mid(M, \gamma) \models \square^{n} \varphi\right\} \subseteq \Delta$. We prove that this is not the case by showing that for any $n$ there is a $\varphi_{n}$ such that

$$
\left(\gamma \models \square^{n} \varphi_{n} \text { and } \omega \models \neg \varphi_{n}\right)
$$

To establish the statement, take $\varphi_{n}=\left(\diamond \square \perp \rightarrow \square^{n} \perp\right)$. We observe that, for all $n$

$$
\begin{aligned}
& \gamma \models \square^{n}\left(\diamond \square \perp \rightarrow \square^{n} \perp\right) \\
& \omega \models \neg\left(\diamond \square \perp \rightarrow \square^{n} \perp\right)
\end{aligned}
$$

To see this, use $x \models \nabla \square \perp \Leftrightarrow x \in \mathbb{N} \cup\{\omega\}$ and $\forall x \in \mathbb{N}\left(x \models \square^{n} \perp \Leftrightarrow x<n\right)$. Furthermore none of the negative integers validates $\diamond \square \perp$.

Now we move to the other part of showing that the model is disharmonious. We have to show that if a formula of the form $\square^{*} \varphi$ holds in $\gamma$, then $\varphi$ holds in $\omega$. It is rather difficult to show this in a direct way. Instead we characterize the set of all formulas that hold in $\omega$. The idea of $\omega$ being the limit of the natural numbers suggests that if a formula holds from a natural number upward, then it holds in $\omega$, and vice versa. But why would the set of formulas that hold from a natural number upward be a maximal consistent set? This is because any formula either holds from a certain natural number upward or it does not hold from a certain natural number upward, i.e. the interpretation of any formula is either finite or cofinite (i.e. its complement is finite).

Lemma 5 For every $\varphi$ :

$$
\omega \models \varphi \text { iff } \llbracket \varphi \text { is cofinite }
$$

where $\llbracket \varphi \rrbracket=\{x \in \mathbb{N} \mid x \models \varphi\}$.

Proof First we show that, for every formula $\varphi, \llbracket \varphi \rrbracket$ is finite or cofinite. The proof is by induction on the structure of $\varphi$. For the base case $(\perp)$, negation and conjunction the proof is trivial. For the case of $\square \varphi$ assume that there is an $x \in \mathbb{N}$ such that $x \not \models \varphi$, therefore $y \not \models \square \varphi$ for all $y>x$, and therefore $\llbracket \square \varphi \rrbracket$ is finite. Otherwise $\llbracket \varphi \rrbracket=\mathbb{N}$, and therefore $\llbracket \square \varphi \rrbracket=\mathbb{N}$, which is cofinite. The case for $\square^{*}$ follows from the previous cases by observing that $\llbracket \square^{*} \varphi \rrbracket=\llbracket \varphi \wedge \square \varphi \rrbracket$, since $R_{c}$ is transitive on $\mathbb{N}$.

Now we prove the statement of the lemma, also by induction on the structure of $\varphi$. Again the cases for $\perp$, negation, and conjunction are easy. Suppose $\omega \models \square \varphi$. This is equivalent to $\llbracket \varphi \rrbracket=\mathbb{N}$, which is equivalent to $\llbracket \square \varphi \rrbracket$ is cofinite, as we saw in the reasoning above. The case for $\llbracket \square^{*} \varphi \rrbracket$ again follows from the previous cases.

Theorem 2 The canonical model for ancestral logic does not have modal harmony: we have $\left(\square^{*}\right)^{-1} \Gamma \subseteq \Delta$ but $(\Gamma, \Delta) \notin R^{*}$.

Proof This follows directly from Lemmas 4 and 5 together with the observation that if $\gamma \models \square^{*} \varphi$, then $\llbracket \varphi \rrbracket=\mathbb{N}$ and therefore $\llbracket \varphi \rrbracket$ is cofinite. Hence $\omega \models \varphi$. 


\subsection{The Case for $\mathrm{PDL}_{\omega}$}

In order to prove the theorem that the canonical model is disharmonious in case of $\mathrm{PDL}_{\omega}$, we use the same countermodel to modal harmony as for the case of ancestral logic. We now consider this to be a model for the language $\mathcal{L}_{0}$ of PDL with an empty set of propositional variables and one atomic program $a$, so $\mathcal{L}_{0}=\mathcal{L}_{\emptyset,\{a\}}$. In this section we prove disharmony for the modality $\left[a^{*}\right]$ and the maximal consistent sets $\Gamma=\left\{\varphi \mid M_{c}, \gamma \models \varphi\right\}$ and $\Delta=\left\{\varphi \mid M_{c}, \omega \models \varphi\right\}$.

As in the case for ancestral logic, we have to prove that $(\Gamma, \Delta) \notin R\left(\alpha^{*}\right)$. The proof of this is completely analogous to the case of ancestral logic. Then we have to prove that a formula is true in $\omega$ iff its extension is cofinite. In order to prove this we need to study the structure of the countermodel in great detail. This is rather technical and it can be skipped by those readers who are not interested in the details of the proof. In order to prove that the model is disharmonious, we do not only have to show that the interpretation of a formula on the natural numbers is finite or cofinite, but we also need a similar (but more complicated) property for programs. We call these sets and relations admissible.

Definition 9 (ADMS and ADMR) The admissible sets ADMS $\subseteq \wp(\mathbb{N})$ are defined as follows.

$$
\operatorname{ADMS}=\bigcup\{\operatorname{ADMS}(n) \mid n \in \mathbb{N}\}
$$

where

$$
\operatorname{ADMS}(n)=\{X \cup Y \mid X \subseteq[0, n), Y \in\{\emptyset,[n, \omega)\}\}
$$

The admissible relations $A D M R \subseteq \wp(\mathbb{N} \times \mathbb{N})$ are defined as follows.

$$
\operatorname{ADMR}=\bigcup\{\operatorname{ADMR}(n, k, p) \mid n \in \mathbb{N}, k \in[1, \omega], p \in[n, \omega)\}
$$

where $\operatorname{ADMR}(n, k, p)$ is the collection

$$
\begin{aligned}
\{T \cup D \cup I(k, n) \cup L(n, p, f) \mid T \subseteq T(n), D \in\{\emptyset, D(n)\}, \\
f:[0, n) \rightarrow([n, p] \cup\{\omega\})\}
\end{aligned}
$$

and

$$
\begin{array}{ll}
T(n) & =\{(x, y) \mid n>x \geq y\} \\
D(n) & =\{(x, x) \mid x \geq n\} \\
I(k, n) & =\{(x, y) \mid x \geq y+k, y \geq n\} \\
L(n, p, f) & =\{(x, y) \mid x \geq f(y), n>y\}
\end{array}
$$

where $x+\omega=\omega$.

Observe that the admissible sets ADMS are simply the finite and cofinite subsets of the natural numbers. See Fig. 2 for a picture of a typical $\operatorname{ADMR}(n, k, p)$. We will show 


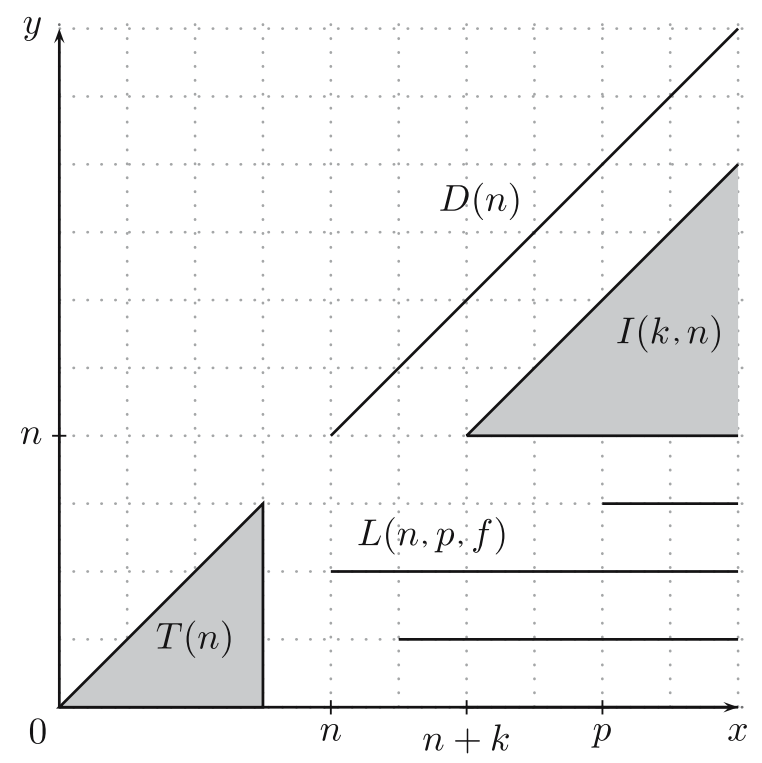

Fig. 2 A typical element of ADMR

that the interpretation of every formula and every program is admissible. But first we prove an auxiliary lemma concerning ADMR.

Lemma 6 ADMR is closed under composition, union, and reflexive transitive closure.

Proof Composition To show that $R \circ R^{\prime} \in \mathrm{ADMR}$ given that $R, R^{\prime} \in \mathrm{ADMR}$, we argue as follows. First note that

$$
\begin{aligned}
& \operatorname{ADMR}(n, k, p) \subseteq \operatorname{ADMR}(n+1, k, \max (p, n+k)) \\
& \operatorname{ADMR}(n, k, p) \subseteq \operatorname{ADMR}(n, k, p+1)
\end{aligned}
$$

Given that $R, R^{\prime} \in \mathrm{ADMR}$, we may assume that there are $n, p$ and $k, k^{\prime}$ such that $R=T \cup D \cup I(k, n) \cup L(n, p, f) \in \operatorname{ADMR}(n, k, p)$ and $R^{\prime}=T^{\prime} \cup D^{\prime} \cup I\left(k^{\prime}, n\right) \cup$ $L\left(n, p, f^{\prime}\right) \in \operatorname{ADMR}\left(n, k^{\prime}, p\right)$ with $T, T^{\prime} \subseteq T(n)$ and $D, D^{\prime} \in\{\emptyset, D(n)\}$. The results of the composition of the components $R$ and $R^{\prime}$ are given in the following table (where $f_{k}^{\prime}=\lambda y \cdot f^{\prime}(y)+k$, and $f_{T^{\prime}}=\lambda y \cdot \min \left\{f(z) \mid z T^{\prime} y\right\}$ and $\min (\emptyset)=\omega$ ):

\begin{tabular}{c||c|c|c|c}
$\circ$ & $T^{\prime}$ & $D(n)$ & $I\left(k^{\prime}, n\right)$ & $L\left(n, p, f^{\prime}\right)$ \\
\hline \hline$T$ & $T \circ T^{\prime} \subseteq T(n)$ & $\emptyset$ & $\emptyset$ & $\emptyset$ \\
\hline$D(n)$ & $\emptyset$ & $D(n)$ & $I\left(k^{\prime}, n\right)$ & $L\left(n, p, f^{\prime}\right)$ \\
\hline$I(k, n)$ & $\emptyset$ & $I(k, n)$ & $I\left(k+k^{\prime}, n\right)$ & $L\left(n, p+k, f_{k}^{\prime}\right)$ \\
\hline$L(n, p, f)$ & $L\left(n, p, f_{T^{\prime}}\right)$ & $\emptyset$ & $\emptyset$ & $\emptyset$
\end{tabular}

So we have

$R \circ R^{\prime} \in \operatorname{ADMR}\left(n, k^{\prime \prime}, p+k\right)$ for some $k^{\prime \prime} \in\left\{k, k^{\prime}, k+k^{\prime}\right\}$ 
and we conclude that $R \circ R^{\prime} \in$ ADMR.

Union To see that that $R \cup R^{\prime} \in \mathrm{ADMR}$, we may again assume that there are $n, p$ and $k, k^{\prime}$ such that $R \in \operatorname{ADMR}(n, k, p)$ and $R^{\prime} \in \operatorname{ADMR}\left(n, k^{\prime}, p\right)$. So $R=T \cup$ $D \cup I(k, n) \cup L(n, p, f), R^{\prime}=T^{\prime} \cup D^{\prime} \cup I\left(k^{\prime}, n\right) \cup L\left(n, p, f^{\prime}\right)$ for certain $T, T^{\prime} \subset$ $T(n), D, D^{\prime} \in\{\emptyset, D(n)\}, f, f^{\prime}:[0, n) \rightarrow([n, p] \cup\{\omega\})$ Now, since

$$
\begin{array}{ll}
I(k, n) \cup I\left(k^{\prime}, n\right) & =I\left(\min \left(k, k^{\prime}\right), n\right) \\
L(n, p, f) \cup L\left(n, p, f^{\prime}\right) & =L\left(n, p, \lambda y \cdot \min \left(f(y), f^{\prime}(y)\right)\right)
\end{array}
$$

we see that $R \cup R^{\prime} \in \operatorname{ADMR}\left(n, \min \left(k, k^{\prime}\right), p\right) \subseteq \mathrm{ADMR}$, and we conclude that ADMR is closed under union.

Reflexive transitive closure To see that $R^{*} \in$ ADMR given that there are $n, k, p$ such that $R=T \cup D \cup I(k, n) \cup L(n, p, f) \in \operatorname{ADMR}(n, k, p)$, we observe that $R^{*}=(R \cup D(n))^{*}$, because of reflexivity. So we may assume that $D=D(n)$. Because of the properties of composition given in the table above, we have that $I(k, n) \subseteq R \circ R$. Observe that $I(2 k, n) \subseteq I(k, n)$. Because $L\left(n, p, f^{\prime}\right) \cup L\left(n, p+k, f_{k}^{\prime}\right)=L\left(n, p, f_{k}^{\prime}\right)$ we have $R \cup(R \circ R) \in \operatorname{ADMR}(n, k, p)$. We now see that $R^{m} \in \operatorname{ADMR}(n, k, p)$ for all $m$. Since $\operatorname{ADMR}(n, k, p)$ is finite and closed under finite unions, it is closed under arbitrary unions and we have $R^{*}=\bigcup\left\{R^{m} \mid m \in \mathbb{N}\right\} \in \operatorname{ADMR}(n, k, p) \subseteq$ ADMR.

Now we prove the following.

Lemma 7 For every formula $\varphi \in \mathcal{L}_{0}$ and every program $\alpha \in \mathcal{L}_{0}$

\section{$\llbracket \varphi \rrbracket \in$ ADMS \\ $\llbracket \alpha \rrbracket \in A D M R$}

where $\llbracket \varphi \rrbracket=\{x \in \mathbb{N} \mid x \models \varphi\}$ and $\llbracket \alpha \rrbracket=R_{c}(\alpha) \cap \mathbb{N} \times \mathbb{N}$.

Proof Simultaneous induction on the structure of $\varphi$ and $\alpha$.

Programs: For the base case, the program $a$, observe that $\llbracket a \rrbracket=I(1,0)$. Moreover $T(0)=\emptyset$, therefore, we can take $D=\emptyset$, and $L(0, p, f)=\emptyset$ for every $p$ and $f$. Therefore $\llbracket a \rrbracket \in \operatorname{ADMR}(0,1,2)$.

For programs the case for tests ? $\varphi$ is also simple. The induction hypothesis implies that $\llbracket \varphi \rrbracket \in$ ADMS, therefore there is some $n$ such that $\llbracket \varphi \rrbracket \in \operatorname{ADMS}(n)$. Note that $\llbracket ? \varphi \rrbracket=\{(x, x) \mid x \in \llbracket \varphi \rrbracket\}$. Now we show that $\llbracket ? \varphi \rrbracket \in \operatorname{ADMR}(n, \omega, n)$. For $D$ we take $\{(x, x) \mid n \leq x$ and $x \in \llbracket \varphi \rrbracket\}$, and for $T$ we take $\{(x, x) \mid n>x$ and $x \in \llbracket \varphi \rrbracket\}$. The set $I(\omega, n)$ is empty and we can take $L(n, p, f)$ to be empty by letting $f(x)$ be $\omega$ for all $x \in[0, n)$. Therefore $\llbracket ? \varphi \rrbracket \in$ ADMR.

The cases for sequential composition, nondeterministic choice and iteration follow from the fact that ADMR is closed under composition, union, and reflexive transitive closure. See Lemma 6.

Formulas: For the base case we only have to consider the formula $\perp$ and the program $a$. Now $\llbracket \perp \rrbracket=\emptyset$ and $\emptyset \in \operatorname{ADMS}(0)$.

The induction step for formulas is easy for negations and conjunctions. It is obvious that each $\operatorname{ADMS}(n)$ is closed under complementation and intersection. Consequently the whole set ADMS is (so it is also closed under union). 
In order to finish the proof of Lemma 7 we have to show that $\llbracket\langle\alpha\rangle \varphi \rrbracket \in$ ADMS, given that $\llbracket \alpha \rrbracket \in A D M R$ and $\llbracket \varphi \rrbracket \in$ ADMS. So we may assume by the induction hypothesis that for some $n, k, p, \llbracket \varphi \rrbracket \in \operatorname{ADMS}(n)$ and $\llbracket \alpha \rrbracket \in \operatorname{ADMR}(n, k, p)$, so $\llbracket \alpha \rrbracket=T \cup D \cup I(k, n) \cup L(n, p, f)$ with $T \subseteq T(n)$ and $D \in\{\emptyset, D(n)\}$. Now $\llbracket\langle\alpha\rangle \varphi \rrbracket$ is a subset of the domain of $\llbracket \alpha \rrbracket$. In fact $\llbracket\langle\alpha\rangle \varphi \rrbracket=(\operatorname{dom}(T) \cap \llbracket\langle\alpha\rangle \varphi \rrbracket) \cup$ $(\operatorname{dom}(D(n)) \cap \llbracket\langle\alpha\rangle \varphi \rrbracket) \cup(\operatorname{dom}(I(k, n)) \cap \mathbb{\llbracket}\langle\alpha\rangle \varphi \rrbracket) \cup(\operatorname{dom}(L(n, p, f)) \cap \llbracket\langle\alpha\rangle \varphi \rrbracket)$. We have

$$
\begin{aligned}
& \operatorname{dom}(T) \cap \llbracket\langle\alpha\rangle \varphi \rrbracket \subseteq[0, n) \\
& \operatorname{dom}(D(n)) \cap \llbracket\langle\alpha\rangle \varphi \rrbracket=\llbracket \varphi \rrbracket \cap[n, \omega) \\
& \operatorname{dom}(I(k, n)) \cap \llbracket\langle\alpha\rangle \varphi \rrbracket=\{x \mid x \geq \min (\llbracket \varphi \rrbracket \cap[n, \omega))+k\} \\
& \operatorname{dom}(L(n, p, f)) \cap \llbracket\langle\alpha\rangle \varphi \rrbracket=\{x \mid x \geq \min \{f(y) \mid y \in \llbracket \varphi \rrbracket \cap[0, n)\}\}
\end{aligned}
$$

As a consequence, $\llbracket\langle\alpha\rangle \varphi \rrbracket \in \operatorname{ADMS}(\max (n+k, p)) \subseteq$ ADMS. This concludes the proof of Lemma 7.

Again we show that as far as formulas are concerned, $\omega$ is the limit of the natural numbers, but we simultaneously prove some other properties of $\omega$.

Lemma 8 For every $\varphi \in \mathcal{L}_{0}$ and for all programs $\alpha \in \mathcal{L}_{0}$ :

$$
\begin{gathered}
\omega=\varphi \text { iff } \llbracket \varphi \rrbracket \text { is cofinite } \\
(\omega, y) \in R_{c}(\alpha) \text { iff }\{x \mid(x, y) \in \llbracket \alpha \rrbracket\} \text { is cofinite } \\
(\omega, \omega) \in R_{c}(\alpha) \text { iff }\{x \mid(x, x) \in \llbracket \alpha \rrbracket\} \text { is cofinite }
\end{gathered}
$$

Proof The proof is by induction on the structure of $\varphi$ and $\alpha$ simultaneously. For formulas, the atomic case is trivial and the cases for negation and conjunction follow directly from Lemma 7. In the case for modal formulas $[\alpha] \varphi$ we take the dual formula $\langle\alpha\rangle \varphi$. For the induction hypothesis, suppose that (5),(6), and (7) hold for $\varphi$ and $\alpha$. It follows from the semantics that $\omega \models\langle\alpha\rangle \varphi$ is equivalent with

$$
\exists y \in \mathbb{N}\left((\omega, y) \in R_{c}(\alpha) \text { and } y \models \varphi\right) \text { or }\left((\omega, \omega) \in R_{c}(\alpha) \text { and } \omega \models \varphi\right)
$$

By the induction hypotheses this is equivalent with

$\exists y \in \mathbb{N}(\{x \mid(x, y) \in \llbracket \alpha \rrbracket\}$ is cofinite and $y \models \varphi$, or $\{x \mid(x, x) \in \llbracket \alpha \rrbracket\}$ is cofinite and $\llbracket \varphi \rrbracket$ is cofinite

It is clear that this implies that $\llbracket\langle\alpha\rangle \varphi \rrbracket$ is cofinite. In the first case the set of worlds that can reach $y$ is cofinite. In the second case the set of worlds where $\varphi$ holds that can reach themselves is cofinite.

The converse implication follows from properties of ADMR. One mainly needs the property that if the domain of $\alpha$ is cofinite, then its intersection with $D(0)$ (i.e. $\{x \mid(x, x) \in \llbracket \alpha \rrbracket\})$ is cofinite, or there is a world $y$ such that $\{x \mid(x, y) \in \llbracket \alpha \rrbracket\}$ is cofinite. This ends the argument for (5).

For (6), the case from right to left with $\alpha=\beta ; \gamma$ is the most complicated. Assume $\{x \mid(x, y) \in \llbracket \beta ; \gamma \rrbracket\}$ is cofinite, therefore the domain of $\llbracket \beta \rrbracket$ is cofinite. 
If $\{x \mid(x, y) \in \llbracket \gamma \rrbracket\}$ finite, then there must be a $z \in \mathbb{N}$ such that $\{x \mid(x, z) \in \llbracket \beta \rrbracket\}$ and $(z, y) \in R_{c}(\gamma)$, therefore we can conclude $(\omega, y) \in R_{c}(\beta ; \gamma)$ using the induction hypothesis (6) for $\beta$. On the other hand if $\{x \mid(x, y) \in \llbracket \gamma \rrbracket\}$ is cofinite, then it follows from the induction hypothesis (6) for $\gamma$ that $(\omega, y) \in R_{c}(\gamma)$. If $\{x \mid(x, x) \in \llbracket \beta \rrbracket\}$ is cofinite, then from the induction hypothesis (7) for $\beta$ it follows that $(\omega, y) \in R_{c}(\beta ; \gamma)$. Otherwise there is a $z \in \mathbb{N}$ such that $\{x \mid(x, z) \in \llbracket \beta \rrbracket\}$ is cofinite and we can find one such that $(z, y) \in R_{c}(\gamma)$. Therefore also in this case $(\omega, y) \in R_{c}(\beta ; \gamma)$ using the induction hypothesis for $\beta$.

The proof of (7) is not too difficult and we omit the details here.

Theorem 3 The canonical model for $\mathrm{PDL}_{\omega}$ does not have modal harmony.

Proof Analogous to the proof of Theorem 2.

The countermodel presented above is not reflexive, transitive, symmetric or euclidean, etc. So proving modal disharmony for logics that have such model restrictions is not as simple as in the case of ancestral logic or $\mathrm{PDL}_{\omega}$. We have not investigated this issue any further.

\section{Conclusion}

In this paper we presented a rather short and elegant proof for the strong completeness of PDL, which is definitely a step forward with respect to proofs given before, as it exploits the hitherto unrevealed identity between saturated and maximal consistent sets. We are not aware of a shorter proof. We generalized the method to a class of countably axiomatized modal logics, containing e.g. epistemic logic with common knowledge. We expected that, like formula harmony, the property of modal harmony would be required for the canonical model of PDL $\omega$. To our surprise, it was not needed, and not even true. Although we came up with a countermodel rather quickly, the subtlety of the arguments required for the $\mathrm{PDL}_{\omega}$ case was also unexpected. It would be interesting to have some kind of fully harmonious canonical model for $\mathrm{PDL}_{\omega}$, but we have not found one yet.

There still remain some issues that may be investigated further. Firstly, it would be interesting to know whether strong completeness for $\mathrm{PDL}_{\omega}$ can be proved with algebraic methods in general and the Rasiowa-Sikorski lemma in particular. This lemma states

Lemma 9 (Rasiowa-Sikorski) Let $\left\{X_{n} \mid n \in \mathbb{N}\right\}$ be a collection of subsets of Boolean algebra $B$ such that each has a meet $\bigwedge X_{n}$ in $B$, and let $a \in B-\{0\}$. Then there is an ultrafilter $F$ of $B$ that contains $a$ and respects every $\wedge X_{n}$ (i.e. satisfies $\left.\forall n \in \mathbb{N}\left(X_{n} \subseteq F \Rightarrow \bigwedge X_{n} \in F\right)\right)$.

(In the original reference Rasiowa and Sikorski (1950), this property is formulated dually in terms of prime ideals, sums and $a \neq 1$.) We investigated this issue in discussion with an anonymous referee, but we have not found a satisfying answer yet.

Secondly, we have not yet found a formulation of the modal harmony property in more mathematical terms, e.g. involving some continuity notion of the modal operators. We refer in this context to Trnkova and Reiterman (1980), where it is shown 
that the modal operators $\langle\alpha\rangle$ are order-continuous in the logic PDL extended with the converse operator - $^{-}$with $R\left(\alpha^{-}\right)=\{(w, v) \mid v R(\alpha) w\}$, while this is not the case for PDL itself. See also Sect. 5.6 of Harel et al. (2000).

Thirdly, propositional dynamic logic and epistemic logic with common knowledge are examples where the introduction of an infinitary rule can be used to attain strong completeness, although the logics are not semantically compact. It should be investigated whether it is possible to characterize the class of non-compact logics where the introduction of infinitary rules leads to strong completeness. This seems to be a hard question, so partial results may be of interest. The general approach of Goldblatt (1993) seems to be a good starting point. In the future we hope to attain similar results for logics with uncountably many rules. This would yield strongly complete proof systems for many more logics of interest.

Acknowledgements We thank Wim Hesselink for discussions on disharmony, and Erik Krabbe, Wiebe van der Hoek and several anonymous referees for their comments on earlier versions of this paper.

\section{References}

Goldblatt, R. (1982). Axiomatising the logic of computer prorgamming. Lecture Notes in Computer Science, Vol. 130. Berlin: Springer-Verlag.

Goldblatt, R. (1993). Mathematics of modality. CSLI Lecture Notes, Vol. 43. Stanford: CSLI Publications.

Harel, D. (1984). Dynamic logic. In D. Gabbay, \& F. Guenthner (Eds.), Handbook of philosophical logic (Vol. II, pp. 497-604). Dordrecht: D. Reidel Publishing Company.

Harel, D., Kozen, D., \& Tiuryn, J. (2000). Dynamic logic. Foundation of computing. Cambrigde: MIT Press.

Knijnenburg, P. M. W. (1988). On axiomatisations for propositional logics of programs. Technical report, University of Utrecht, Nov 1988. RUU-CS-88-34.

Knijnenburg, P. M. W., \& van Leeuwen, J. (1991). On models for propositional dynamic logic. Theoretical Computer Science, 91, 181-203.

Kooi, B. P. (2003). Knowledge, chance, and change. $\mathrm{PhD}$ thesis, Department of Mathematics and Computing Science, University of Groningen.

Kooi, B., Renardel de Lavalette, G., \& Verbrugge, R. (2006). Hybrid logics with infinitary proof systems. Journal of Logic and Computation, 16, 161-175.

Kozen, D., \& Parikh, R. (1981). An elementary proof of the completeness of PDL. Theoretical Computer Science, 14, 113-118.

Mirkowska, G. (1981). PAL-Propositional algorithmic logic. In E. Engeler (Ed.), Logic of programs. Lecture notes in computer science, (Vol. 125, pp. 23-101). Berlin: Springer-Verlag.

Pratt, V. R. (1976). Semantical considerations on Floyd-Hoare logic. In Proceedings of the 17th IEEE Symposium on the Foundations of Computer Science, pp. 109-112.

Rasiowa, H., \& Sikorski, R. (1950). A proof of the completeness theorem of Gödel. Fundamenta Mathematicae, 37, 193-200.

Renardel de Lavalette, G. R., Kooi, B. P., \& Verbrugge, R. (2002). Strong completeness for propositional dynamic logic. In P. Balbiani, N.-Y. Suzuki, \& F. Wolter (Eds.), AiML2002-Advances in modal logic (conference proceedings) (pp. 377-393). Institut de Recherche en Informatique de Toulouse IRIT.

Salwicki, A. (1970). Formalized algorithmic languages. Bulletin de l'Académie Polonaise des Sciences: Série des sciences mathématiques, astronomiques et physiques, 18, 227-232.

Segerberg, K. (1982). A completeness theorem in the modal logic of programs. In T. Traczyck (Ed.), Universal Algebra and Applications. Papers presented at the Seminar held at the Stefan Banach International Mathematical Center 1978, (Vol. 9, pp. 31-46). PWN, Warsaw: Banach Center Publications.

Segerberg, K. (1994). A model existence theorem in infinitary propositional modal logic. Journal of Philosophical Logic, 23, 337-367.

Trnkova, V., \& Reiterman, J. (1980). Dynamic algebras which are not Kripke structures. In P. Dembiński (Ed.), Proceedings of the 9th Symposium on Mathematical Foundations of Computer Science. Lecture notes in computer science, (Vol. 88, pp. 528-538). Berlin: Springer-Verlag. 